\title{
Valence Focus and Self-Esteem Lability: Reacting to Hedonic Cues in the Social Environment
}

\author{
Paula R. Pietromonaco \\ University of Massachusetts at Amherst
}

\author{
Lisa Feldman Barrett \\ Boston College
}

\begin{abstract}
Individuals differ in the extent to which they emphasize feelings of pleasure or displeasure in their verbal reports of emotional experience, termed valence focus (VF). Two event-contingent, experience-sampling studies examined the relationship between VF and sensitivity to pleasant and unpleasant social cues. It was predicted, and found, that individuals with greater VF (i.e., who emphasized feelings of pleasure/ displeasure in reports of emotional experience) demonstrated greater self-esteem lability (i.e., larger changes in self-esteem) to pleasant and unpleasant information contained in social interactions than did those lower in VF. These effects held even after statistically controlling for possible confounding variables (neuroticism, affect intensity). Implications for understanding the psychological impact of valenced interpersonal events are discussed.
\end{abstract}

Keywords: valence focus, emotion, self-esteem lability, experience sampling, social interactions

Hedonic tone (pleasure and displeasure), also called valence, is a fundamental property of the mind (Barrett, 2006a). It is a basic building block of emotional life in humans (Barrett, 2006b; Ortony \& Turner, 1990; Russell, 2003; Russell \& Barrett, 1999). Objective measurements used in the study of human emotion-such as peripheral nervous system activation (Bradley \& Lang, 2000; Cacioppo, Berntson, Klein, \& Poehlmann, 1997, Cacioppo, Berntson, Larsen, Poehlmann, \& Ito, 2000), facial movements (Cacioppo et al., 1997, 2000; Messinger, 2002; Russell, Bachorowski, \& Fernández-Dols, 2003), vocal acoustics (Bachorowski, 1999), expressive behavior (Cacioppo \& Gardner, 1999), and neural activations (Barrett \& Wager, 2006) — all give evidence of valenced affect (pleasure or displeasure) or its intensity. This basic affect system not only forms the core of emotion but also grounds other psychological phenomena, including attitudes (e.g., Cacioppo \& Berntson, 1994; Eagly \& Chaiken, 1998; Ito \& Cacioppo, 2001), stereotyping and prejudice (e.g., Cacioppo \& Berntson, 2001; Forgas \& Fiedler, 1996; Moreno \& Bodenhausen, 2001), verbal communication and negotiation (e.g., Forgas, 1998, 1999a, 1999b), moral judgment and decision making (e.g., Forgas, 1995; Haidt, 2002; Slovic, Finucane, Peters, \& MacGregor, 2002), work motivation (e.g., Seo, Barrett, \&

Paula Pietromonaco, Department of Psychology, University of Massachusetts, Amherst; Lisa Feldman Barrett, Department of Psychology, Boston College, Chestnut Hill, Massachusetts.

Preparation of this article was supported by a National Institutes of Health Director's Pioneer Award (DP1OD003312), a National Institute of Mental Health Independent Scientist Research Award (K02 MH001981), NIA Grant R01 AG030311, and National Science Foundation Grants SBR-9727896 and BCS 0527440 to Lisa Feldman Barrett.

Correspondence concerning this article should be addressed to Paula Pietromonaco, Department of Psychology, 135 Hicks Way, Tobin Hall, University of Massachusetts, Amherst, MA 01003-9271; or to Lisa Feldman Barrett, Department of Psychology, 427 McGuinn Hall, Boston College, Chestnut Hill, MA 02467. E-mail: monaco@psych.umass.edu
Bartuneck, 2004), health (Gallo, Bogart, Vranceanu, \& Matthews, 2005), psychopathology (e.g., R. J. Davidson, 2000; R. J. Davidson et al., 2002), well-being (e.g., R. J. Davidson, 2004), personality (e.g., Revelle, 1995; Watson, 2000; Yik, Russell, Ahn, Dols, \& Suzuki, 2002), and memory (Kensinger \& Schacter, in press). It is also a core property of consciousness (Edelman \& Tononi, 2000; Searle, 2004; for a discussion, see Duncan \& Barrett, 2007).

Virtually everyone can tell the difference between a pleasant feeling and an unpleasant feeling, and people communicate these feelings both implicitly (Barrett, 1998, 2004; Feldman, 1995) and explicitly in their self-reports of emotional experience (Barrett \& Russell, 1998; Russell \& Barrett, 1999; Russell, Weiss, \& Mendelsohn, 1989; for a review, see Barrett, 2006a). Yet there is significant variation across individuals in the extent to which people attend to and emphasize hedonic content in their reports of emotional experience, termed valence focus (VF; Barrett, 1998, 2004; Feldman, 1995). Valence focus represents the amount of information about pleasure or displeasure contained in verbal reports of emotional experience. Individuals high in VF emphasize pleasure and displeasure in the content of their verbal reports more than do those lower in VF. When people high in VF rate emotion adjectives to report their experience of emotion, they primarily emphasize pleasure and displeasure, whereas people low in VF take into account valence to a lesser extent during the rating process. For some people, valence is the only aspect of experience on which they focus (i.e., they are highly valence focused). These individuals use emotion terms such as angry, sad, and nervous for what they have in common (to indicate feeling negative). For others, valence is one of many aspects that describe their experience (i.e., they are lower in VF); these individuals use the same emotion terms to report their experience, but in a way that emphasizes the distinctiveness in the words, thereby focusing on other properties of experience as well (e.g., arousal).

A person's degree of VF is related to his or her sensitivity to affectively potent information in the environment. Individuals who are largely valence focused in their reports of experience charac- 
terize themselves as more sensitive to both reward and punishment cues than are those lower in VF. In comparison with individuals lower in VF, individuals high in VF described themselves as more neurotic, extraverted, and affectively intense, as well as having greater behavioral inhibition and activation (Barrett, 2006a). Furthermore, individuals with greater VF also show greater perceptual sensitivity to changes in facial affect (Barrett \& Niedenthal, 2004). Specifically, when individuals completed a "morph movies" task in which each trial showed a face with a neutral expression that gradually shifted to a clear emotional expression, participants higher in VF displayed greater perceptual sensitivity to facial affect by detecting the onset of unpleasant facial expressions much earlier than did those lower in VF. Thus, it is likely that VF shapes reactions and behavior largely by increasing attention to hedonic cues in the environment. Although the effects of VF can arise in any context, the current work focuses on the role of VF within a social context because interpersonal interactions are particularly likely to provide emotionally evocative and self-relevant cues.

\section{Valence Focus and Self-Esteem Lability}

For individuals with enhanced perceptual sensitivity to information of affective value, slight changes in facial behavior or tone of voice during a social interaction will have greater psychological meaning and greater consequences for their immediate well-being. A larger number of people and situations will be appraised as having value (i.e., as having the potential to be helpful or harmful in a given instant) and therefore have an enhanced opportunity to impact the person's momentary state. Inhabiting a world that is filled with frequent, salient valenced events will lead not only to a greater number of perturbations in a person's hedonic state, but we would predict that it would also lead to larger and more frequent momentary changes in self-esteem (or how the person evaluates his or her own worth at a given moment in time). That is, we would expect to see a link between VF and self-esteem lability.

Self-esteem lability is defined as changes in self-esteem in response to specific events or cues (Barnett \& Gotlib, 1988) and captures the continuing relationship between precipitating events and resulting evaluations of the self across time (Butler, Hokanson, \& Flynn, 1994). A central component of the definition of lability is that self-esteem fluctuates in response to specific, momentary events in the immediate situation. Although little work has examined changes in momentary self-esteem that occur in response to immediate events, some studies have investigated whether selfesteem experienced at the end of the day covaries with reports of the average number of positive or negative events on that day. Consistent with the idea that self-esteem varies as a function of experiences, this work has found that people show higher daily self-esteem on days when they report more positive events and lower daily self-esteem on days when they report more negative events (Nezlek, 2005; Nezlek \& Plesko, 2003). The present work builds on this earlier work by examining whether people show fluctuations in momentary self-esteem immediately after experiencing positive or negative events. Furthermore, for an event to impact momentary self-esteem, it must be judged as having some value or import to well-being. Thus, this work examines whether individuals who are higher in VF and who are more perceptually sensitive to the valenced cues that other people convey through various channels (face, body, and speech) are particularly likely to demonstrate enhanced self-esteem lability in response to specific social interactions.

Self-esteem lability is distinct from the closely related concept of self-esteem stability (e.g., Kernis, 2005). Self-esteem lability focuses on fluctuations in self-esteem that occur in response to specific, immediate situational cues and is measured as the covariation of changes in self-esteem in the presence of a specific cue (e.g., the size of a regression coefficient). In contrast, self-esteem stability refers to the variance in self-esteem ratings (regardless of what might have caused them) and is measured as the size of the standard deviation in self-esteem ratings over time or instances. Of course, self-esteem stability and lability can be related to one another. In one study (Greenier et al., 1999), the standard deviation in self-reports of self-esteem taken every $12 \mathrm{hr}$ for 5 days was related to self-esteem changes when participants recalled and reported on their most positive event and most negative event at the end of each day; those with more unstable self-esteem reported feeling worse about themselves after their most important (recalled) negative event and feeling better about themselves after their most important (recalled) positive event to a greater extent than did those with more stable self-esteem. The present work differs from this prior study because it examines moment-tomoment changes in self-esteem in response to specific positive and negative cues that occur within social interactions rather than changes in self-esteem that result from recalled events of a general hedonic nature.

Furthermore, in considering the connection between VF and self-esteem lability, it is important to emphasize that the two constructs are distinct. Specifically, VF represents the amount of information about pleasure or displeasure contained in verbal reports of emotional experience. It is not a measure of variability in emotional experience, and it does not represent the tendency to report pleasant states or unpleasant states. Instead, VF reflects the extent to which hedonic valence is an important descriptive property of core affective responding for a given individual. Individuals high in VF emphasize pleasure and displeasure in the content of their verbal reports more than do those lower in VF, often at the expense of other properties of affect, such as arousal (Barrett, 2004). So, unlike self-esteem lability, VF is not a measure of variability; rather, it is the kind of information that accounts for variability.

In the present work, we investigated whether enhanced VF was associated with enhanced self-esteem lability in two eventcontingent sampling studies. Participants reported on their immediate, online perceptions of positive and negative information, their momentary self-esteem, and their momentary experience of emotion after every social interaction that they engaged in for 1 week (Study 1) or 2 weeks (Study 2). As in prior studies, VF was computed as a behavioral index of the proportion of variance accounted for by valence in the participant's sample of selfreported emotional experiences. VF reflects the patterns of selfreport ratings (e.g., the covariance in ratings of "angry" and "sad") across multiple occasions and settings, and it represents the extent to which participants' patterns of responses are characterized by positivity or negativity (i.e., valence). Thus, the computation of VF treats self-reports as instances of verbal behaviors, and it focuses on the pattern of these verbal behaviors rather than on the level or magnitude of any single rating or the explicit content of the self-reported experience of emotion. Furthermore, VF can be con- 
sidered an implicit measure because participants do not explicitly report on how much they focus on positive or negative feelings; instead, VF reflects the proportion of variance in each person's sample of self-report ratings that is accounted for by the valencebased meanings of the words. Thus, the measure of VF indicates the extent to which the patterning within participants' self-reports of emotional experience reveals their emphasis on the hedonics of their experience, as is indicated by their emphasis on the valencebased meaning of the words during the reporting process.

In both studies, participants reported on their daily social interactions using a pencil-and-paper version of the Rochester Interaction Record (Reis \& Wheeler, 1991). The data were collected in 1994 (Study 1) and 1996 (Study 2), prior to the widespread use of electronic devices (e.g., personal digital assitants, or PDAs) to capture responses. Researchers have debated the relative merits of paper versus electronic diary methods, but recent work (Green, Rafaeli, Bolger, Shrout, \& Reis, 2006) suggests that findings based on paper diary reports often parallel those produced by electronic reports. Furthermore, in our studies, we instituted a number of procedures to increase compliance (e.g., establishing a collaborative relationship with participants) and to assess the degree to which participants followed the instructions (e.g., postexperimental questions that encouraged participants to honestly report on the percentage of time that they completed diary records from memory).

We hypothesized that individuals higher in VF would display more highly labile self-esteem when compared with those who showed lower VF. Specifically, we reasoned that high VF individuals would be more sensitive to situations containing threat or reward cues, and these situations therefore would be more evocative for them, thereby leading to more intense changes in selfesteem. Thus, we hypothesized that VF would moderate the association between experiencing a hedonic social cue and self-esteem. For the purposes of discriminant validity, we also examined the link between self-esteem lability and arousal focus (AF, or the extent to which self-reports of experience contain information about feelings of activation and deactivation). Furthermore, we also examined whether any link between VF and self-esteem lability could be accounted for by other, related personality variables. Both neuroticism (Costa \& McCrae, 1992) and affect intensity (Larsen, Diener, \& Emmons, 1986) are related to, but not quite identical with, the concept of VF. Neuroticism is a multifaceted construct that includes the propensity to experience negative emotional states and to have vulnerable self-esteem. Affect intensity is a construct that represents the characteristic intensity or strength with which an individual reports pleasant and unpleasant emotional experience over time. Individuals who are highly valenced focused describe themselves as higher in neuroticism and affect intensity (Barrett, 2006b), but prior work (Barrett \& Niedenthal, 2004) has shown that the link between VF and perceptual sensitivity to affect is not accounted for by these variables. Thus, we expected that VF would remain significantly related to self-esteem lability even after taking into account neuroticism or affect intensity.

\section{Study 1}

The purpose of Study 1 was to test the hypothesis that individuals higher in valence focus would show greater changes in self- esteem as a function of positive and negative cues from their social interaction partners. The Study 1 data came from an eventcontingent experience-sampling study in which participants provided ratings of their feelings about themselves, their partners' responses, and their affective reactions immediately after every social interaction that lasted for $10 \mathrm{~min}$ or longer over a 1-week period.

\section{Method}

\section{Participants}

Participants were 70 undergraduate psychology students (27 of whom were men) at the University of Massachusetts, Amherst, or at Pennsylvania State University who had complete data for the interaction record ratings and reported using memory to complete their interaction records no more than $30 \%$ of the time. Average age of the participants in the final sample was $19.23(S D=1.45)$. All participants received course credit and tickets for a $\$ 50$ lottery for their participation. Full details regarding the sample can be found in Pietromonaco and Barrett (1997). ${ }^{1}$

\section{Measures}

Interaction record. Only the variables relevant to this report are discussed. The procedure for collecting the interaction record data followed the format described by Reis and Wheeler (1991). Immediately after every social interaction lasting for $10 \mathrm{~min}$ or longer, participants rated the degree of conflict, and the extent to which their interaction partner expressed both positive and negative emotion during the interaction. Participants also rated how worthwhile, competent, and accepted they had felt during (or immediately after) each social interaction. Ratings were made on 5-point scales ranging from not worthwhile (1) to worthwhile (5); incompetent (1) to competent (5); and not accepted by your partner (1) to accepted by your partner (5). These ratings were summed to yield an index of momentary self-esteem.

In addition, participants described their affective reactions during the interaction using adjectives that sampled all eight octants of the affective circumplex (enthusiastic, excited, happy, satisfied, calm, relaxed, quiet, tranquil, bored, dulled, sad, disappointed, nervous, angry, surprised, and energetic). Ratings for each adjective were made on 5-point scales ranging from not at all (1) to a great deal (5). These ratings were used to compute an index of VF (the proportion of variance in emotion ratings accounted for by valence) according to standard published procedures (for details, see below; also see Barrett, 1998, 2004, 2006a; Barrett \& Niedenthal, 2004; Feldman, 1995). In addition, we computed AF, or the extent to which people emphasize arousal when reporting their experiences over time. AF can be thought of as the amount of information about activation and deactivation contained in verbal reports of experiences of emotion given over time (Barrett, Quig-

\footnotetext{
${ }^{1}$ The data used in Study 1 came from an archived data set that has been previously used to examine adult attachment patterns (Pietromonaco \& Barrett, 1997), the intimacy process (Laurenceau, Barrett, \& Pietromonaco, 1998), and sex differences in emotion (Barrett, Robin, Pietromonaco, \& Eyssell, 1998). The hypotheses under investigation here do not overlap in any way with those in previously published reports.
} 
ley, Bliss-Moreau, \& Aronson, 2004). Self-reports characterized by high AF contain a lot of information about activation and deactivation, whereas those characterized by low AF contain less of this information.

Rosenberg self-esteem scale. During the initial session, participants completed this 10-item measure of global self-esteem (Rosenberg, 1965) as part of a larger set of questionnaires. Items (e.g., "On the whole, I am satisfied with myself" and "I am able to do things as well as most other people") were rated on a 4-point scale $(0=$ strongly agree and $4=$ strongly disagree $)$. For this sample, the mean was $22.47(S D=5.89)$, and Cronbach's alpha reliability was .92 .

Neuroticism and affect intensity. To determine whether any effects of valence focus might be accounted for by variance shared with the conceptually related self-report measures of neuroticism and affect intensity, participants also completed measures of both of these constructs. The neuroticism scale (48 items) of the Revised NEO Personality Inventory (NEO PI-R; Costa \& McCrae, 1992) is a standard measure that has repeatedly shown good reliability and validity. Participants rated the neuroticism items on a 5-point scale $(1=$ strongly disagree, $3=$ neutral, $5=$ strongly agree $)$. Participants also completed the Affect Intensity Measure (AIM; Larsen et al., 1986). This measure includes 40 items such as "When something good happens, I am usually much more jubilant than others" and "When I do feel anxiety it is normally very strong." Participants rated the items on a scale that ranged from 1 (never) to 6 (always).

\section{Procedure}

Participants attended three laboratory sessions. During the first session, participants completed a battery of self-report measures. The only measure from this battery that was relevant for the present work was the Rosenberg Self-Esteem Scale. Research assistants provided participants with verbal and written instructions on the use of the interaction protocol. Participants returned their interaction records three times during their recording week, and they received extra lottery tickets for returning their forms on time. The experimenter phoned, within $24 \mathrm{hr}$, any participants who did not return their forms on time and reminded them to return the forms. During the third laboratory session, the experimenter interviewed participants about their compliance (in particular, the percentage of interaction forms that they had completed from memory). The experimenter stressed that participants would not be penalized in any way (i.e., they would still receive credit and lottery tickets) if they had not followed instructions and that we were simply interested in obtaining accurate information about their data (for additional details, see Pietromonaco \& Barrett, 1997). Participants also reviewed a list of all of their interaction partners during the recording week and indicated their relationship to them (e.g., romantic partner, friend, acquaintance, or roommate), the length of their relationship, and the overall closeness of the relationship with the interaction partner. ${ }^{2}$

To compute VF, we directly estimated the amount of variance in the ratings of affective experience accounted for by hedonic valence. Note that although participants provide ratings of their emotional experiences, the degree to which they emphasize hedonics is estimated as the proportion of variance accounted for in the set of their self-report ratings. Thus, participants do not directly report on how much hedonics matter to them; instead, VF is a more indirect, behavioral measure of the amount of hedonic content contained in self-report ratings of emotional experience. In operational terms we ask, "How much of the correlation between ratings of two emotional experiences accounted for by the valencebased similarity of the words?" For example, sadness is characterized as an unpleasant state that is low in activation, and anxiety as an unpleasant state that is high in activation; the two words are similar in terms of the valence they denote, but different in arousal. If a person sometimes reports feeling both anxious and sad, and other times feels one but not the other, this will result in a correlation of zero because the person is rating anxiety and sadness in distinct ways, using both valence-based and arousal-based information in the words' meanings to represent his or her experiences of emotion. In contrast, if a person consistently reports both together (or neither) at every measurement instance, this will produce a correlation of 1 because the person is using the words to represent what the states have in common (i.e., valence) and ignoring how they differ (i.e., arousal).

Specifically, to assess VF, we first computed a P-correlation matrix for each participant (i.e., the correlation in ratings of different emotional experiences across time). It is possible to estimate how much a person emphasizes valence in the rating of his or her experience by correlating each P-correlation matrix with external criteria indicating the valence similarity of the words. The external criterion used here was the cognitive structure of emotion language (e.g., the valence-based meaning of the emotion words used in the rating process). We correlated the P-correlation matrix for the self-reports to the valence-based cognitive structure of the word to estimate the proportion of variance due to valence in the self-report ratings of experience for each individual (also see Barrett, 1998 , 2004; Barrett \& Niedenthal, 2004; Barrett et al., 2004; Feldman, 1995). If the valence-based similarity of the words accounts for a large proportion of variance in the correlations between ratings of emotional experiences for a given person, then this person is high in VF. If the valence-based similarity of the words accounts for a small proportion, then the person is low in VF. This way of computing valence focus does not reflect whether participants are primarily reporting positive or negative states per se. A similar procedure was followed to assess AF. For more details about how to compute VF and AF, see Feldman (1995) and Barrett (1998, 2004).

Valence focus indices ranged from $r=-.03$ to $r=.77$, with a mean of $r=.41(S D=.17)$. AF indices ranged from $r=.05$ to $r=.86$, with a mean of $r=.38(S D=.16)$. As in prior studies, $\mathrm{VF}$ and AF were negatively related $(r=-.54, p<.001)$.

\section{Results and Discussion}

The complete data set consisted of 2,311 interactions from 70 participants. Participants returned an average of 33.01 records $(S D=13.4)$; VF and AF indices were not related to the total number of interactions recorded $(r=.12, p=.34$, for VF and $r=$

\footnotetext{
${ }^{2}$ We examined whether partner closeness moderated the effects, but it did not do so reliably across the two studies. In Study 1, people higher in valence focus showed lower self-esteem when conflict occurred, and this association was even stronger when the interaction partner was a close other. This effect was not replicated in Study 2.
} 
$-.05, p=.69$, for AF). Neither VF nor AF were related to scores on the Rosenberg Self-Esteem Scale, suggesting that the tendency to experience valenced (or arousing) affect was not related to generalized positive or negative beliefs about the self $(r=.06, p=$ .61 , and $r=-.10, p=.39$, for $\mathrm{VF}$ and AF, respectively).

Self-esteem lability was indexed as the magnitude of the relationship between evocative events (e.g., the amount of conflict in a social interaction) and self-esteem ratings, and it was estimated as the size of a regression coefficient (i.e., how much conflict predicts a change in self-esteem across occasions for one person). Larger coefficients indicate greater lability. We used a random coefficient multilevel modeling procedure (Hierarchical Linear Modeling, or HLM; HLM version 6.03; Raudenbush, Bryk, Cheong, \& Congdon, 2004) to estimate the magnitude of selfesteem lability in response to each specific social cue (e.g., conflict) for each participant, the variability in lability estimates across individuals, and the extent to which VF accounted for that variability. HLM analyses using only VF and AF as predictors of momentary self-esteem indicated that neither VF nor AF were related to the mean level of self-esteem across all interactions, although people varied considerably from one another in their mean levels of momentary self-esteem, $\chi^{2}(67)=932.48, p<.001$.

For the main analyses, all models contained a Level 1 predictor (e.g., the amount of conflict in a social interaction), a Level 1 criterion (i.e., momentary rating of self-esteem), a Level 1 control variable (i.e., a lagged self-esteem variable to control for the self-esteem level during the prior interaction), and two Level 2 predictors (VF and AF). Level 1 predictors were centered around the group mean, and Level 2 predictors were centered around their grand mean. The lagged self-esteem variable was not a significant predictor in any of the Study 1 analyses. Furthermore, because neither VF nor AF moderated the size of the lagged self-esteem variable in any analysis, the analyses reported here did not include $\mathrm{VF}$ and $\mathrm{AF}$ as moderators of the lagged self-esteem variable.

We also examined whether $\mathrm{VF}$ and $\mathrm{AF}$ were associated with variance in any of the Level 1 predictors (i.e., conflict, perceptions that the partner expressed negative emotion, and perceptions that the partner expressed positive emotion). VF was unrelated to reported levels of conflict or perceptions that partners expressed positive emotion, although people higher in VF reported that their partners expressed more negative emotion, $b=.839, t(67)=2.15, p=.035$. For this reason, it was important to center Level 1 predictors around the group mean. AF was not significantly related to any of the predictors.

\section{Self-Esteem Lability to Negative Events}

Conflict. The results of the HLM analyses appear in Table 1. As conflict increased in a social interaction, self-esteem became significantly more negative. As was predicted, VF significantly moderated this effect, indicating that participants higher in VF showed greater decreases in self-esteem in response to conflict than did those lower in VF. Figure 1 shows the simple regression lines for the association between conflict and self-esteem at levels of VF 1 standard deviation $(S D)$ below the mean, at the mean, and $1 S D$ above the mean. In line with the hypothesis, the relationship (i.e., the slope) between conflict and self-esteem was stronger for individuals higher in VF than for those lower in VF. Individuals who were high in VF showed the greatest change in self-esteem $(b=-.85)$. The association was attenuated for individuals who evidenced a moderate level of VF $(b=-.58)$, and it was even weaker for those low in VF $(b=-.32)$. Arousal focus did not significantly moderate the association between self-esteem and conflict.

Perceived partner expression of negative emotion. Table 1 shows that, as interaction partners were perceived to express more negative emotion in a social interaction, self-esteem became significantly more negative. VF appeared to moderate this relationship, but the effect did not reach the conventional level of significance. The relationship (i.e., the slope) between conflict and self-esteem tended to be stronger for individuals higher in VF than for those lower in VF, which is in line with the prediction. Individuals who were high in VF showed the largest self-esteem lability $(b=-.65)$, whereas those moderate in VF showed less $(b=-.50)$, and those low in VF showed the least $(b=-.35)$. AF did not significantly moderate this association.

\section{Self-Esteem Lability to Perceived Partner Expression of Positive Emotion}

As interaction partners were perceived to express increasingly more positive emotion during a social interaction, self-esteem became significantly more positive. Although VF did not significantly moderate this association, the effect was in the predicted direction (see Table 1). Individuals higher in VF showed the strongest positive change in self-esteem in response to this positive cue $(b=.84)$ when compared with those moderate in $\operatorname{VF}(b=.70)$ or low in VF $(b=.56)$. As was expected, AF was not related to self-esteem lability, $b=.206, S E=.469, t(67)=.44, p=.662 .^{3}$

\section{Analyses Controlling for Neuroticism and Affect Intensity}

To determine whether the effects of VF might be accounted for by potentially related variables (i.e., neuroticism and affect intensity), we reanalyzed the data including neuroticism as an additional Level 2 predictor in one set of analyses and affect intensity as an additional Level 2 predictor in another set. The findings were very similar to those reported in the main analyses.

Neuroticism. We observed a double dissociation when both VF and neuroticism were entered as Level 2 predictors in our HLM analyses: Neuroticism predicted the mean level of selfesteem across all interactions (the size of the intercept in the HLM analyses) but did not predict self-esteem lability (the size of the regression coefficient that reflects change in self-esteem in response to a specific valenced cue); VF continued to predict selfesteem lability, but it did not predict the mean level of self-esteem. For example, in the analyses including level of conflict as a predictor, neuroticism did not predict self-esteem lability in response to conflict, $b=-.003, t(64)=-.96, p=.341$, but did predict a lower average level of self-esteem across all interactions, $b=-.013, t(64)=-2.00, p=.049$. Neuroticism did not moderate self-esteem lability when individuals perceived that their partner expressed negative emotion, $b=-.000, t(64)=-.16, p=.875$, or

\footnotetext{
${ }^{3}$ Although we did not expect any gender differences, we conducted analyses including gender and the interactions between gender and valence focus and gender and arousal focus. Gender did not moderate any of the predicted effects in either study.
} 
Table 1

Study 1: Valence Focus as a Moderator of the Relationship Between Hedonic Cues and Momentary Self-Esteem

\begin{tabular}{|c|c|c|c|c|c|}
\hline Variable & $b$ & $t(67)$ & $p$ & $M$ & $S D$ \\
\hline DV & - & - & - & 13.30 & 2.33 \\
\hline \multicolumn{6}{|c|}{ Negative cue: Conflict } \\
\hline VF & .594 & .47 & $n s$ & .41 & .17 \\
\hline $\mathrm{AF}$ & .703 & .61 & $n s$ & .38 & .16 \\
\hline Conflict & -.584 & -6.99 & .001 & 1.64 & 1.01 \\
\hline Conflict $\times V F$ & -1.588 & -2.55 & .014 & - & - \\
\hline Conflict $\times \mathrm{AF}$ & -.466 & -.73 & $n s$ & - & - \\
\hline \multicolumn{6}{|c|}{ Negative cue: Partner expression of negative emotion } \\
\hline VF & .654 & .51 & $n s$ & - & - \\
\hline $\mathrm{AF}$ & .708 & .60 & $n s$ & - & - \\
\hline PNE & -.504 & -6.78 & .001 & 1.96 & 1.17 \\
\hline $\mathrm{PNE} \times \mathrm{VF}$ & -.895 & -1.45 & .153 & - & - \\
\hline $\mathrm{PNE} \times \mathrm{AF}$ & -.661 & -.83 & $n s$ & - & - \\
\hline
\end{tabular}

Positive cue: Partner expression of positive emotion

\begin{tabular}{|c|c|c|c|c|c|}
\hline VF & .626 & .48 & $n s$ & - & - \\
\hline $\mathrm{AF}$ & .684 & .57 & ns & - & - \\
\hline PPE & .702 & 11.63 & .001 & 3.49 & 1. \\
\hline $\mathrm{PPE} \times \mathrm{VF}$ & .831 & 1.64 & .105 & - & - \\
\hline $\mathrm{PPE} \times \mathrm{AF}$ & .206 & .44 & $n s$ & - & - \\
\hline
\end{tabular}

Note. $\mathrm{DV}=$ momentary self-exteem $\mathrm{VF}=$ valence focus $; \mathrm{AF}=$ arousal focus; $\mathrm{PNE}=$ partner negative emotion; $\mathrm{PPE}=$ partner positive emotion; $n s=$ not significant.

when they perceived that their partner expressed positive emotion, $b=.001, t(64)=.62, p=.535$, but neuroticism predicted average self-esteem across all interactions in both cases: partner negative emotion, $b=-.013, t(64)=-2.04, p=.045$; partner positive emotion, $b=-0.012, t(64)=-1.92, p=.058$.

Affect intensity. A similar double-dissociation was observed between VF and affect intensity. When affect intensity was included in the analyses, VF continued to predict the degree of self-esteem lability but did not predict mean levels of self-esteem across all analyses. Affect intensity did not significantly predict self-esteem lability to conflict, $b=-0.133, t(64)=-1.19, p=$ .238 , perceptions of the partner's negative emotion, $b=-.010$, $t(64)=-.11, p=.916$, or perceptions of the partner's positive emotion, $b=.085, t(64)=.80, p=.428$, but predicted, at marginally significant levels, higher average self-esteem for analyses, including conflict, $b=.465, t(64)=1.75, p=.085$, perceptions of the partner's negative emotion, $b=.472, t(64)=$ $1.78, p=.080$, and perceptions of the partner's positive emotion, $b=.464, t(64)=1.75, p=.085$; in contrast, VF did not predict average levels of self-esteem in any of these analyses (all $p$ s $>$ $.68)$.

Overall, Study 1 provides some support for the hypothesis that individuals higher in VF show more self-esteem lability in response to positive and negative cues from their interaction partners, although only the analysis predicting conflict reached a conventional level of significance. Furthermore, we observed, as was predicted, that the effects were specific to VF; VF, but not AF, moderated the association between self-esteem and the valenced social cues. In addition, the link between VF and self-esteem lability cannot be accounted for by neuroticism or affect intensity. We further tested the hypothesis using data from a different event-contingent study that included ratings for a larger number of interactions (across a 2-week sampling period) and assessed more types of valenced cues from the partner.

\section{Study 2}

In Study 2, we further tested the hypothesis using a larger number of items to explicitly assess the perception of positive and negative cues from the interaction partner. The study also included a more neutral cue (i.e., the partner talked about his or her thoughts or facts), allowing us to examine whether VF contributed to self-esteem lability selectively for valenced cues. In addition, participants in Study 2 completed interaction diaries over a longer sampling period (14 days) than the one used in Study 1, thus

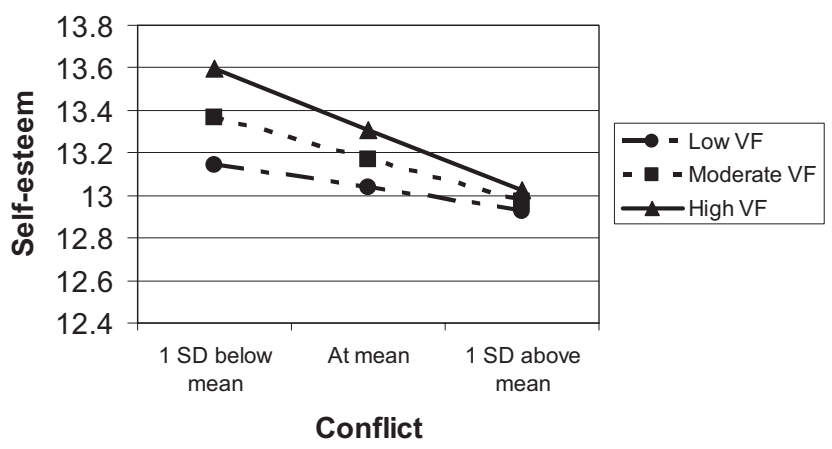

Figure 1. Study 1: Association between conflict and self-esteem at different levels of valence focus (VF). 
enabling us to examine the predicted processes across a greater number of interactions.

\section{Method}

\section{Participants}

The final sample included the 64 undergraduate students (14 of whom were men) at Boston College who had complete data and who reported using memory less than $30 \%$ of the time. Average age of the final sample was 20.8 years $(S D=6.96)$. Participants received extra credit in psychology courses as well as tickets for a chance to win prizes (e.g., Boston College hats and sweatshirts) at the end of the study.

\section{Interaction Record}

The interaction record was similar in format to the one used in Study 1, with the exception that it included several different items relevant to the hypothesis that people high in VF would show greater self-esteem lability in response to threat or reward cues from their partner. Participants rated three negative (potentially threatening) aspects of their interactions on a 5-point scale: the degree to which the partner expressed negative emotion $(1=$ not at all and $5=a$ great deal), the degree to which the partner criticized them $(1=$ not at all and $5=$ a great deal $)$, and the degree to which the partner and participant disagreed with each other $(1=$ not at all and $5=$ for most of the interaction). Participants also rated two positive (potentially rewarding) aspects of the interaction: the degree to which the partner expressed positive emotion and the degree to which the partner liked or approved of them during or immediately following the interaction (for all items, $1=$ not at all and $5=$ a great deal). In addition, participants rated a more neutral cue from the partner: the degree to which the partner talked about his or her thoughts or facts $(1=$ not at all and $5=$ a great deal). Participants rated their momentary self-esteem using three 5-point scales (not worthwhile/worthwhile, incompetent/competent, and unacceptable/acceptable).

Participants also completed affective ratings after each interaction, and these ratings were used to compute $\mathrm{VF}$ and $\mathrm{AF}$ indices following the method described in Study 1. The adjectives were the same as those used in Study 1 except that "nervous" was replaced with "afraid." Valence focus indices ranged from $r=.32$ to $r=1.00$, with a mean of $r=.63(S D=.14)$. Arousal focus indices ranged from $r=.01$ to $r=.70$, with a mean of $r=.25(S D=.15)$. As in prior studies, VF and AF were negatively associated $(r=-.38$, $p<.01)$.

\section{Procedure}

The data collection procedure followed the same format used in Study 1 except that participants completed Rochester Interaction Record forms for 2 weeks (14 days) rather than for 1 week (7 days).

\section{Results and Discussion}

The data set for Study 2 consisted of 3,551 interactions from 64 participants. Participants returned an average of 55.48 ( $S D=$ 23.48) records; $\mathrm{VF}$ and $\mathrm{AF}$ indices were not related to the total number of interactions recorded $(r=-.06, p=.67$, for $\mathrm{VF}$ and $r=.03, p=.79$, for $\mathrm{AF}$ ). Neither $\mathrm{VF}$ nor $\mathrm{AF}$ were related to scores on the Rosenberg Self-Esteem Scale (for this sample, $M=$ $21.75, S D=6.00)$, suggesting that the tendency to experience valenced (or arousing) affect was not related to generalized positive or negative beliefs about the self $(r=-.06, p=.62$, for VF and $r=.00, p=.99$, for $\mathrm{AF}$ ).

As in Study 1, neither VF nor AF were related to the mean level of momentary self-esteem across all interactions (estimated with intercept-only HLM analyses), but people varied considerably from one another in their mean levels of momentary self-esteem, $\chi^{2}(61)=2043.43, p<.001$. In contrast to Study 1 , the lagged self-esteem variable frequently was significant in the Study 2 analyses, indicating that changes in self-esteem often persisted across interactions. Furthermore, the magnitude of this effect varied considerably, indicating that individual differences existed in the extent to which self-esteem changes persisted over time. However, neither VF nor AF moderated the size of the lagged selfesteem variable in any analysis.

Most of the predictor variables were marginally related to VF and AF. Higher VF was associated marginally with less disagreement, $b=-1.094, t(61)=-1.76, p=.083$, and less perceived partner criticism, $b=-0.962, t(61)=-1.68, p=.098$. Also, $\mathrm{VF}$ was associated significantly with less perceived partner expression of negative emotion, $b=-1.498, t(61)=-2.12, p=.038$. In addition, higher AF was associated marginally with less perceived partner expression of negative emotion, $b=-1.042, t(61)=$ $-1.83, p=.072$, and more desire to gain the partner's approval, $b=.937, t(61)=1.77, p=.082$. These findings indicate that individuals higher in VF were not interpreting events as more valenced when compared with those lower in VF. Similarly, individuals higher in $\mathrm{AF}$, for the most part, were not interpreting events as more arousing than those lower in AF. However, to control for these associations, Level 1 predictors were group centered.

\section{Self-Esteem Lability to Negative Events}

Consistent with the hypothesis, VF predicted greater self-esteem lability for each of the three perceived negative events (partner expression of negative emotion, partner criticism, and partner and self disagreed). Unexpectedly, AF also moderated self-esteem lability in two of the three analyses.

Perceived partner disagreement. Just as VF moderated the association between self-esteem and conflict in Study 1, it also moderated the association between self-esteem and the degree to which individuals perceived disagreement with their partner. Table 2 shows that, as partners were perceived to disagree more, selfesteem became more negative, and VF significantly moderated this relationship. Figure 2 shows the simple regression lines for individuals who were $1 S D$ above the mean, at the mean, and $1 S D$ below the mean in valence focus. As was predicted, individuals high in VF showed the strongest drop in self-esteem when the partners were perceived to disagree more $(b=-.44)$; this association was weaker for those moderate in VF $(b=-.27)$ and weakest for those low in VF $(b=-.10)$. The relationship between self-esteem and partner disagreement also varied significantly as a function of AF. Individuals high in $\mathrm{AF}(\mathrm{b}=-.40)$, in comparison 
Table 2

Study 2: Valence Focus as a Moderator of the Relationship Between Hedonic Cues and Momentary Self-Esteem

\begin{tabular}{|c|c|c|c|c|c|}
\hline Variable & $b$ & $t(61)$ & $p$ & $M$ & $S D$ \\
\hline DV & - & - & - & 12.81 & 2.64 \\
\hline \multicolumn{6}{|c|}{ Negative cue: Partners disagreed } \\
\hline VF & -1.152 & -.56 & $n s$ & .63 & .13 \\
\hline $\mathrm{AF}$ & -.012 & -.02 & $n s$ & .25 & .15 \\
\hline Disagreement & -.269 & -4.40 & .001 & 1.72 & 1.04 \\
\hline Disagree $\times \mathrm{VF}$ & -1.267 & -2.15 & .035 & - & - \\
\hline Disagree $\times \mathrm{AF}$ & -.916 & -2.17 & .034 & - & - \\
\hline \multicolumn{6}{|c|}{ Negative cue: Partner expression of negative emotion } \\
\hline VF & -1.287 & -.629 & $n s$ & - & 一 \\
\hline $\mathrm{AF}$ & -.037 & -.025 & $n s$ & - & - \\
\hline PNE & -.129 & -2.55 & .014 & 2.49 & 1.26 \\
\hline $\mathrm{PNE} \times \mathrm{VF}$ & -.923 & -2.06 & .044 & - & - \\
\hline $\mathrm{PNE} \times \mathrm{AF}$ & -.893 & -2.65 & .011 & - & 一 \\
\hline
\end{tabular}

\begin{tabular}{|c|c|c|c|c|c|}
\hline \multicolumn{6}{|c|}{ Negative cue: Partner criticized } \\
\hline VF & -1.193 & -.585 & ns & & \\
\hline $\mathrm{AF}$ & .02 & .013 & ns & & \\
\hline $\mathrm{PC}$ & -.492 & -5.99 & .001 & 1.37 & .83 \\
\hline $\mathrm{PC} \times \mathrm{VF}$ & -1.454 & -2.71 & .009 & & \\
\hline $\mathrm{PC} \times \mathrm{AF}$ & -1.003 & -1.54 & .13 & & \\
\hline
\end{tabular}

\begin{tabular}{|c|c|c|c|c|c|}
\hline \multicolumn{6}{|c|}{ Positive cue: Partner expression of positive emotion } \\
\hline VF & -1.263 & -.62 & $n s$ & - & - \\
\hline $\mathrm{AF}$ & .063 & .04 & $n s$ & - & - \\
\hline PPE & .467 & 7.60 & .001 & 3.96 & 1.05 \\
\hline $\mathrm{PPE} \times \mathrm{VF}$ & 1.239 & 2.47 & .017 & - & - \\
\hline $\mathrm{PPE} \times \mathrm{AF}$ & .731 & 1.85 & .069 & - & - \\
\hline
\end{tabular}

\begin{tabular}{|c|c|c|c|c|c|}
\hline \multicolumn{6}{|c|}{ Positive cue: Gained liking/approval from partner } \\
\hline $\mathrm{VF}$ & -.846 & -.43 & ns & - & - \\
\hline $\mathrm{AF}$ & .228 & .15 & ns & - & - \\
\hline GL & .585 & 9.86 & .001 & 3.40 & 1.24 \\
\hline GL $\times$ VF & .542 & 1.37 & .176 & - & - \\
\hline $\mathrm{GL} \times \mathrm{AF}$ & -.039 & -.09 & $n s$ & - & - \\
\hline \multicolumn{6}{|c|}{ Neutral cue: Partner talked about facts } \\
\hline VF & -1.263 & -.62 & ns & - & - \\
\hline $\mathrm{AF}$ & .017 & .01 & ns & - & - \\
\hline PTF & .099 & 1.77 & .082 & 4.10 & 1.03 \\
\hline $\mathrm{PTF} \times \mathrm{VF}$ & .151 & .35 & $n s$ & - & - \\
\hline $\mathrm{PTF} \times \mathrm{AF}$ & -.047 & -.14 & ns & - & - \\
\hline
\end{tabular}

Note. $\mathrm{DV}=$ momentary self-esteem $; \mathrm{VF}=$ valence focus; $\mathrm{AF}=$ arousal focus; $\mathrm{PNE}=$ partner negative emotion; $\mathrm{PC}=$ partner criticized; $\mathrm{PPE}=$ partner positive emotion; $\mathrm{GL}=$ gained liking; $\mathrm{PTF}=$ partner talked about facts; $n s=$ not significant.

with those moderate $(\mathrm{b}=-.27)$ or low in $\mathrm{AF}(\mathrm{b}=-.14)$, showed the steepest drop in self-esteem when disagreement occurred.

Perceived partner expression of negative emotion. Selfesteem became more negative during interactions in which partners were perceived to express more negative emotion. Consistent with the prediction, VF significantly moderated this association (see Table 2). Individuals high in VF showed larger decreases in self-esteem during interactions in which they perceived that their partners expressed negative emotion $(b=.25)$ when compared with those moderate in VF $(b=-.13)$. Furthermore, those low in VF showed no change $(b=.01)$ in self-esteem as a function of the partner's expression of negative emotion. This pattern is parallel to the one shown in Figure 2. AF again moderated the link between the perception of the partner's expression of negative emotion and self-esteem. Similar to the pattern for VF, individuals high in AF evidenced a greater decrease in self-esteem for interactions in which partners were perceived to express negative emotion $(b=$ -.27); those moderate in AF showed a similar but smaller decrease $(b=-.13)$; those low in $\mathrm{AF}$ showed no decrease in self-esteem as a function of the partner's perceived expression of negative emotion $(b=.01)$.

Perceived partner criticism. Similarly, when individuals perceived greater partner criticism, they showed lower self-esteem, and in line with the hypothesis, this association was moderated by VF (see Table 2). High VF participants evidenced the greatest drop in self-esteem when they perceived that their partner expressed 


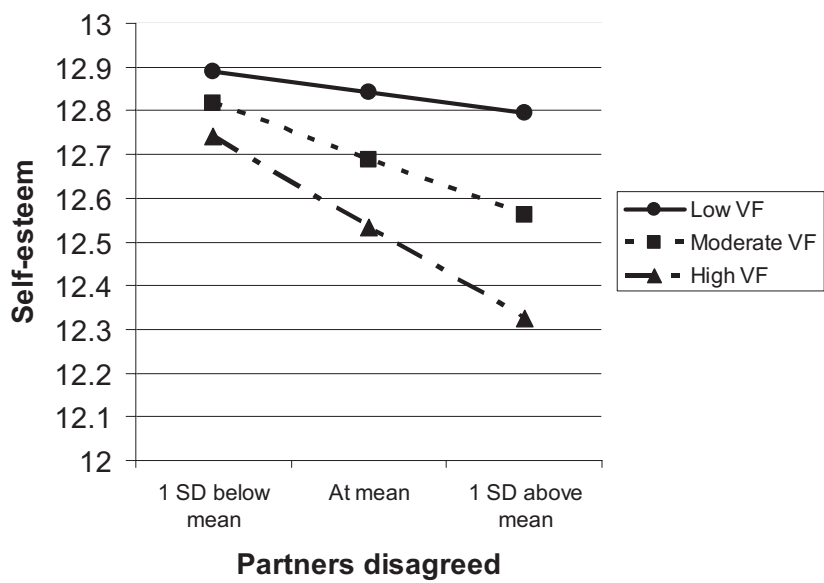

Figure 2. Study 2: Association between partner disagreement and selfesteem at different levels of valence focus (VF).

criticism ( $b=-.69)$; moderate VF participants showed a similar but less sharp drop $(b=-.49)$; and low VF participants evidenced an even smaller drop in self-esteem $(b=-.30)$. As is indicated in Table 2, AF did not significantly moderate this association, although the effect was in the same direction as the effects reported for the other two negative cues.

\section{Self-Esteem Lability to Positive Events}

Consistent with the hypothesis, VF predicted greater self-esteem lability in response to one positive interpersonal cue (interactions in which the partner was perceived to express positive emotion), but it did not predict self-esteem changes in response to perceptions that the partner expressed liking or approval. AF showed patterns similar to those for VF.

Perceived partner expression of positive emotion. When partners were perceived as expressing more positive emotion, participants reported higher self-esteem, and this effect was moderated significantly by VF (see Table 2). Individuals high in VF showed the greatest increase in self-esteem when they reported that their partners expressed positive emotion $(b=63)$; those moderate in VF showed a moderate increase $(b=.47)$, and those low in VF showed the least increase ( $b=.30$; see Figure 3 ). AF marginally moderated the association between the partner's positive expression of emotion and self-esteem. The pattern was similar to the one for VF: Self-esteem increased with perceptions that the partner expressed positive emotion, and this increase was most pronounced for those high in $\mathrm{AF}(b=.58)$ and least pronounced for those low in $\operatorname{AF}(b=.47)$ and in between these two groups for those moderate in $\mathrm{AF}(b=.36)$.

Gained liking or approval from the partner. When participants reported that they had gained more liking or approval from the interaction partner, they also showed an increase in self-esteem (see Table 2). This association was not moderated significantly by $\mathrm{VF}$, although the effect was in the predicted direction. AF also did not moderate this association.

\section{Self-Esteem Lability to Neutral Events}

The degree to which the partner was perceived to talk about facts was expected to be neutral (i.e., not valenced), and thus VF was not expected to moderate the association between this variable and self-esteem. Although individuals who perceived that the partner expressed thoughts showed marginally higher self-esteem, neither VF nor AF significantly moderated this association (see Table 2). Importantly, these findings demonstrate that the body of findings as a whole cannot be explained as simply some individuals having more variability in their ratings (and therefore showing larger regression coefficients as a consequence).

\section{Analyses Controlling for Neuroticism and Affect Intensity}

All of the effects reported in the main analyses remained significant when either neuroticism or affect intensity were included in the analyses, and neither neuroticism nor affect intensity significantly moderated the associations between self-esteem and any of the positive or negative social cues. As in Study 1, we found a double dissociation between VF and neuroticism. For example, when predicting the association between perceived partner disagreement and self-esteem, VF remained a significant moderator, $b=-1.251, t(60)=-2.11, p=.039$, but neuroticism did not moderate this association, $b=-.080, t(60)=-.67, p=.508$. In contrast, neuroticism predicted lower average levels of selfesteem, $b=-1.394, t(60)=-3.25, p=.002$, whereas VF did not, $b=-0.896, t(60)=-.50, p=.621$. Thus, VF (and not neuroticism) predicted self-esteem lability in the presence of valenced cues, whereas neuroticism (and not VF) predicted average levels of self-esteem. When affect intensity was included in the analysis, VF continued to significantly moderate all effects reported as significant in the main analyses; affect intensity, however, did not significantly moderate the association between any of the predictors and self-esteem. Furthermore, unlike the findings in Study 1 , affect intensity did not predict mean levels of self-esteem in any analyses.

Study 2 findings generally support the hypothesis that people who are valence focused show greater fluctuations in momentary self-esteem in response to valenced cues from their social interaction partners, and they replicate and extend the findings from Study 1 . When individuals detected negative cues from the partner (disagreement, criticism, and partner expression of negative emo-

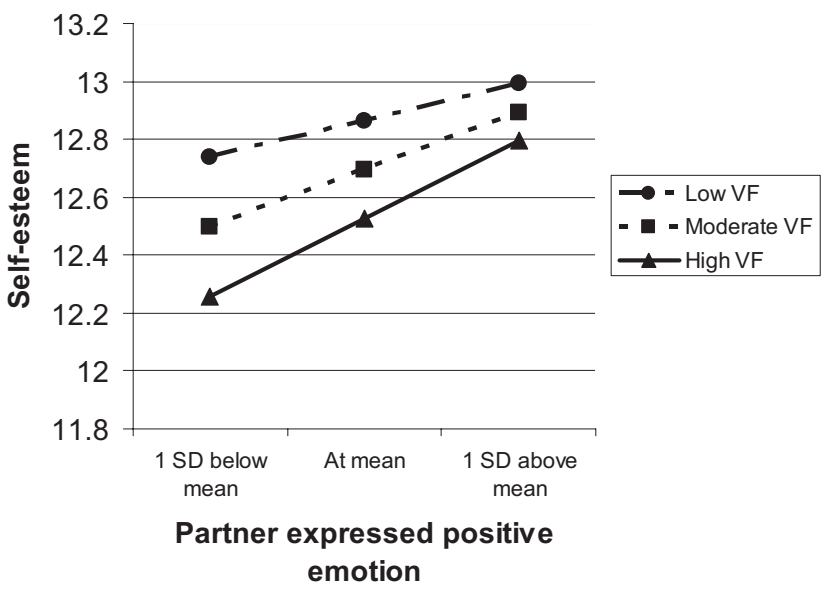

Figure 3. Study 2: Association between perceived partner expression of positive emotion and self-esteem at different levels of valence focus (VF). 
tion), individuals higher in VF evidenced more marked declines in self-esteem than did those lower in VF, replicating the effect found in Study 1 for interactions that included greater conflict. Furthermore, we found that in contexts in which partners were perceived to express positive emotion, individuals who were higher in VF evidenced a greater increase in self-esteem than did those lower in VF. The pattern for this positive cue replicated the trend found in Study 1 for the same positively valenced cue. However, the predicted moderating effect of VF did not emerge as clearly for another positive cue, perceived approval from the partner, possibly because some people may not have interpreted liking or approval as a positive interpersonal cue. Other work suggests that individuals who emphasize self-reliance and who are averse to depending on others (e.g., those higher in dismissing/avoidance; Bartholomew \& Horowitz, 1991) may even interpret this cue as negative because it could imply a dependence on the responses of others. Thus, variability in how participants interpreted the receipt of liking or approval may have attenuated the effect.

The Study 2 findings also indicated that VF did not moderate the association between self-esteem and a neutral cue (the partner talking about facts). This finding supports the idea that the moderating effect of VF applies specifically to valenced cues in the current situational context. In addition, we unexpectedly found in Study 2 that individuals higher in AF showed greater self-esteem lability in response to perceived negative and positive cues, and this moderating effect paralleled the one for VF. Finally, the predicted effects held even when possible confounding variables (neuroticism and affect intensity) were included in the analyses, and neither neuroticism nor affect intensity significantly predicted lability in self-esteem in the face of valenced social cues.

\section{Mini Meta-Analysis}

To examine the reliability of the moderating effect of VF on the link between valenced cues and self-esteem across the two studies, we performed a mini meta-analysis. We used the Stouffer method (Mosteller \& Bush, 1954; Rosenthal, 1984) to assess the combined $p$ levels for the three variables (conflict/disagreement, partner's perceived expression of negative emotion, and partner's perceived expression of positive emotion) that were included in both studies. Across the two studies, VF significantly moderated the associations between conflict and self-esteem, $Z=3.24, p=.001$, one-tailed; the partner's perceived expression of negative emotion and self-esteem, $Z=2.42, p=.008$, one-tailed; and the partner's perceived expression of positive emotion and self-esteem, $Z=$ $2.81, p=.003$, one-tailed. Overall, the two studies provide reliable support for the hypothesis that individuals higher in VF show greater self-esteem lability in response to valenced cues.

\section{General Discussion}

Some individuals live a life of drama and are easily moved or perturbed by changes in their surroundings, while others live a life of tranquility, and they are generally unaffected by the vicissitudes of life. In two event-contingent experience-sampling studies, we have demonstrated that VF (the extent to which self-reports of emotion contain a lot of information about pleasure and displeasure, or hedonics) predicts this variation. As was expected, across both studies, individuals who evidenced higher VF showed signif- icantly greater self-esteem lability to negative aspects of social interactions when compared with those lower in VF. Those individuals who emphasized the pleasantness or unpleasantness of their emotional experience showed stronger drops in their selfesteem when they perceived threatening social cues such as conflict, disagreement, criticism, or a partner's expression of negative emotion. In addition, across both studies, individuals higher in VF showed greater increases in self-esteem when they perceived a rewarding cue (the partner's expression of positive emotion). These findings are consistent with the hypothesis that individuals high in VF are more responsive to the valenced social cues that they encounter in their daily lives than are individuals lower in VF. Furthermore, the greater sensitivity of high VF individuals appears to be specific to valenced cues; the self-esteem of high VF individuals did not change more than that of low VF individuals in response to neutral social cues. In addition, the moderating effect of VF was evident even after controlling for potentially related personality variables (neuroticism and affect intensity), providing further support that VF predicts outcome measures over and above measures of emotional reactivity (Barrett \& Niedenthal, 2004; Barrett et al., 2004). Interestingly, we observed a double dissociation between neuroticism and VF in both studies. Neuroticism predicted individuals' average levels of self-esteem (i.e., an average perception of the self across all kinds of interactions) in both studies, but VF did not. In contrast, VF predicted changes in self-esteem as a function of hedonic cues perceived in the environment, but neuroticism did not. Thus, neuroticism seems to be associated with a more general or average perception of the self, whereas VF predicts the variations that occur in self-esteem in reaction to hedonic cues.

Because VF was assessed in an implicit manner (i.e., calculated from the proportion of variance in each person's sample of ratings that is accounted for by the valence-based meanings of the words), it is unlikely that participants attempted to respond in a manner consistent with their expectations about VF. In measuring VF, participants did not explicitly report that they were high or low in VF; instead, their degree of VF was computed from the covariation in their self-report ratings of emotion words across multiple interactions, partners, and settings. Thus, individuals who implicitly evidenced a pattern of highly valenced responses were more likely to show changes in their self-esteem in response to positive or negative social cues.

These findings suggest that individuals who experience strong, valenced emotions tend to be more sensitive to their social environment and that this sensitivity may produce greater fluctuations in their feelings about themselves. Prior work (Barrett \& Niedenthal, 2004) has shown that high valence-focused individuals more quickly perceived changes in negative facial expressions, and this greater perceptual sensitivity was not accounted for by other variables such as neuroticism, extraversion, and need for evaluation. The present studies extend this work by demonstrating that the greater sensitivity of high valence-focused individuals is present in their everyday perceptions in a more natural social interaction context. Furthermore, the findings show that individuals who are high in VF are more attuned to both negatively and positively valenced social cues and that their reactivity to those cues impacts their momentary feelings about themselves.

Barring certain forms of mental illness, people are sensitive to their social environment to some degree. However, enhanced 
sensitivity to environmental cues is considered a risk factor for depression (Barnett \& Gotlib, 1988), suggesting that individuals high in VF may be at higher risk for mood disorders such as depression. Support for this idea comes from research (Butler et al., 1994) that has demonstrated that depressed individuals or those with a prior history of depression evidenced higher self-esteem lability than did those who had never been depressed. Furthermore, individuals who showed high self-esteem lability and who experienced significant life stress were more likely to develop depression (Butler et al., 1994). Additional work also has shown that people whose self-worth is contingent on external cues evidence poorer psychological adjustment (e.g., Crocker, Luhtanen, Cooper, $\&$ Bouvrette, 2003) and are more vulnerable to depression (Sargent, Crocker, \& Luhtanen, 2006). This prior work, together with the present findings, suggests that individuals who are high in VF, who show greater self-esteem lability in response to externally available threat and reward cues, also may be more prone to develop depression.

These ideas raise the possibility that VF may serve as a risk factor for some forms of psychopathology. VF is an indicator of variations in affective responding, and such variations have been linked to several forms of psychopathology, including Axis I disorders-such as various forms of anxiety, major depression, and schizophrenia - and some Axis II disorders-such as borderline personality disorder. Variations in affective responding may predispose people to alcohol and drug dependence and may signal a brain's proneness to psychopathology, such that certain variations can be considered transdisorder vulnerabilities. Many psychological disorders share a common or "transdiagnostic" (Harvey, Watkins, Mansell, \& Shafran, 2004) disturbance in affective processing (Barlow, 2002; Kring, in press) that involves vigilance to threat (Quigley \& Barrett, 1999), is on a continuum with normal personality variability (Weinstock \& Whisman, 2006), and has been associated with the short allele of a serotonin transporter (5-HTT) promoter polymorphism, which is thought to produce an affective vulnerability to environmental stress (Hariri \& Holmes, 2006). One question for future research is whether VF represents an identifiable risk factor for these transdisorder variations in affective responding.

Our findings also converge with recent work (Zeigler-Hill \& Showers, 2007) on self-esteem stability that has compared individuals who organize information about themselves into distinct positively and negatively valenced aspects (i.e., who compartmentalize self-aspects) with those who integrate positive and negatively valenced aspects (i.e., who integrate self-aspects). Individuals who compartmentalized evaluative self-aspects were more likely than those who integrated evaluative self-aspects to show a greater increase in state self-esteem on days when they reported experiencing more positive events and a greater decrease in state self-esteem on days when they reported experiencing more negative events. Although the methodology of the Zeigler-Hill and Showers (2007) study differed in important ways from the methodology in the present work (e.g., they assessed the stability of self-esteem from day to day rather than momentary self-esteem lability in response to specific cues; self-esteem and daily events were assessed retrospectively at the end of each day rather than immediately after events occurred), compartmentalized individuals showed patterns similar to those found in the present work for individuals high in VF. Future work might examine whether high
VF individuals' greater sensitivity to affective cues in the environment shapes the nature of their self-structure, leading them to develop more evaluatively compartmentalized views of self.

Several limitations need to be considered in interpreting these findings. First, although VF may lead to greater attention to valenced situational cues, thereby resulting in greater ups and downs in self-esteem, the correlational nature of the study cannot rule out bidirectional or third variables that may account for the observed effects. For example, it is possible that having a history of fluctuations in self-esteem leads individuals to show greater VF, or that a third variable, such as generalized negative feelings about the self, underlies both greater self-esteem lability and greater VF. In the present studies, we were able to rule out the possibility that generalized negative feelings about the self accounted for the findings; global self-esteem, as assessed by the Rosenberg SelfEsteem Inventory, was not related to either VF or AF. Furthermore, we were able to rule out neuroticism and affect intensity as alternative explanatory variables; in contrast to the effects observed for VF, neither neuroticism nor affect intensity predicted changes in self-esteem when valenced social cues were present.

Second, participants' reports determined whether they experienced a positive social cue or a negative social cue, and the degree to which these subjective perceptions map onto the objective characteristics of the situation is unknown. People who are high in VF may experience a higher base rate of rewarding or threatening cues (although this pattern was generally not evident in Study 1, and the relationships were in the opposite direction in Study 2). This limitation will need to be addressed in further work in which high and low VF individuals respond to a set of objectively designated rewarding and threatening social cues.

Third, a fundamental measurement issue relates to the way in which VF and AF were calculated. Both indices were based on correlating each participant's sample of emotion ratings to valence-based and arousal-based semantic matrices. Typically, a correlation coefficient becomes a good estimator of the population correlation coefficient only when sample sizes are large. In the present study, the sample size of emotion ratings varied widely across participants, with some participants having fairly small samples. As a result, the VF and AF indices might be better estimates of the true level of VF and AF for participants who had a larger number of interactions than for those who had a smaller number. Because measurement error reduces the magnitude of the regression coefficient and attenuates the size of estimates of interaction effects, it may be that individuals with smaller sample sizes had lower VF estimates and therefore smaller regression coefficients and less self-esteem lability. However, this alternative explanation can be ruled out because variations in VF were not related to the number of interactions reported. Furthermore, error (both random and systematic) is not likely to produce spurious interactions (Aiken \& West, 1991). Thus, it is unlikely that the effect of VF on self-esteem lability was spurious because of the error inherent in the estimation of VF.

Finally, VF was consistently and uniquely related to self-esteem lability across both studies, even when AF was unexpectedly predicting the degree of self-esteem lability (in Study 2). Notably, AF was not consistently related to greater shifts in self-esteem when either positive or negative social cues were present. Further work is needed to establish the consistency of the relationship between $\mathrm{AF}$ and self-esteem lability. 
Overall, the present findings demonstrate that pleasure and threat cues in the social environment are more evocative for people who are high in $\mathrm{VF}$, leading them to experience greater momentary fluctuations in their feelings about themselves. These findings invite further investigation to evaluate whether this enhanced sensitivity to affective cues and accompanying self-esteem lability place high VF individuals at greater risk for depression and other mood disorders.

\section{References}

Aiken, L. S., \& West, S. G. (1991). Multiple regression: Testing and interpreting interactions. Newbury Park, CA: Sage.

Bachorowski, J. A. (1999). Vocal expression and perception of emotion. Current Directions in Psychological Science, 8, 53-57.

Barlow, D. H. (2002). Anxiety and its disorders: The nature and treatment of anxiety and panic (2nd ed.). New York: Guilford Press.

Barnett, P. A., \& Gotlib, I. H. (1988). Psychological functioning and depression: Distinguishing among antecedents, concomitants, and consequences. Psychological Bulletin, 104, 97-126.

Barrett, L. F. (1998). Discrete emotions or dimensions? The role of valence focus and arousal focus. Cognition and Emotion, 12, 579-599.

Barrett, L. F. (2004). Feelings or words? Understanding the content in self-report ratings of experienced emotion. Journal of Personality and Social Psychology, 87, 266-281.

Barrett, L. F. (2006a). Valence as a basic building block of emotional life. Journal of Research in Personality, 40, 35-55.

Barrett, L. F. (2006b). Solving the emotion paradox: Categorization and the experience of emotion. Personality and Social Psychology Review, 10, $20-46$.

Barrett, L. F., \& Niedenthal, P. M. (2004). Valence focus and the perception of facial affect. Emotion, 4, 266-274.

Barrett, L. F., Quigley, K., Bliss-Moreau, E., \& Aronson, K. R. (2004). Arousal focus and interoceptive sensitivity. Journal of Personality and Social Psychology, 87, 684-697.

Barrett, L. F., Robin, L., Pietromonaco, P. R., \& Eyssell, K. (1998). Are women the more emotional sex? Evidence from emotional experiences in social interactions. Cognition and Emotion, 12, 555-578.

Barrett, L. F., \& Russell, J. A. (1998). Independence and bipolarity in the structure of current affect. Journal of Personality and Social Psychology, 74, 967-984.

Barrett, L. F., \& Wager, T. D. (2006). The structure of emotion: Evidence from neuroimaging studies. Current Directions in Psychological Science, $15,79-83$.

Bartholomew, K., \& Horowitz, L. M. (1991). Attachment styles among young adults: A test of a four-category model. Journal of Personality and Social Psychology, 61, 226-244.

Bradley, M. M., \& Lang, P. J. (2000). Measuring emotion: Behavior, feeling, and physiology. In R. D. Lane \& L. Nadel (Eds.), Cognitive neuroscience of emotion (pp. 242-276). New York: Oxford University Press.

Butler, A. C., Hokanson, J. E., \& Flynn, H. A. (1994). A comparison of self-esteem lability and low trait self-esteem as vulnerability factors for depression. Journal of Personality and Social Psychology, 66, 166-177.

Cacioppo, J. T., \& Berntson, G. G. (1994). Relationship between attitudes and evaluative space: A critical review, with emphasis on the separability of positive and negative substrates. Psychological Bulletin, 115, 401-423.

Cacioppo, J. T., \& Berntson, G. G. (2001). The affect system and racial prejudice. In J. Bargh \& D. K. Apsley (Eds.), Unravelling the complexities of social life: A festschrift in honor of Robert B. Zajonc (pp. 95-110). Washington, DC: American Psychological Association.

Cacioppo, J. T., Berntson, G. G., Klein, D. J., \& Poehlmann, K. M. (1997).
The psychophysiology of emotion across the lifespan. Annual Review of Gerontology and Geriatrics, 17, 27-74.

Cacioppo, J. T., Berntson, G. G., Larsen, J. T., Poehlmann, K. M., \& Ito, T. A. (2000). The psychophysiology of emotion. In R. Lewis \& J. M. Haviland-Jones (Eds.), The handbook of emotion (2nd ed., pp. 173-191). New York: Guilford Press.

Cacioppo, J. T., \& Gardner, W. L. (1999). Emotion. Annual Review of Psychology, 50, 191-214.

Costa, P. T., \& McCrae, R. R. (1992). The Revised NEO Personality Inventory (NEO PI-R) professional manual. Odessa, FL: Psychological Assessment Resources.

Crocker, J., Luhtanen, R. K., Cooper, M. L., \& Bouvrette, A. (2003). Contingencies of self-worth in college students: Theory and measurement. Journal of Personality and Social Psychology, 85, 894-908.

Davidson, R. J. (2000). Affective style, psychopathology, and resilience: Brain mechanisms and plasticity. American Psychologist, 55, 11961214.

Davidson, R. J. (2004). Affective style: Causes and consequences. In J. T. Cacioppo \& G. G. Berntson (Eds.), Essays in social neuroscience (pp. 77-91). Cambridge, MA: MIT Press.

Davidson, R. J., Lewis, D. A., Alloy, L. B., Amaral, D. G., Bush, G., \& Cohen, J. D., et al. (2002). Neural and behavioral substrates of mood and mood regulation. Biological Psychiatry, 52, 478-502.

Duncan, S., \& Barrett, L. F. (2007). The amygdala in visual awareness. Trends in Cognitive Sciences, 11, 190-192.

Eagly, A. H., \& Chaiken, S. (1998). Attitude structure and function. In D. T. Gilbert, S. T. Fiske, \& G. Lindzey (Eds.), The handbook of social psychology (4th ed., Vol. 1, pp. 269-322). New York: McGraw-Hill.

Edelman, G. M., and Tonini, G. (2000). A universe of consciousness: How matter becomes investigation. New York: Basic Books.

Feldman, L. A. (1995). Valence focus and arousal focus: Individual differences in the structure of affective experience. Journal of Personality and Social Psychology, 69, 153-166.

Forgas, J. P. (1995). Mood and judgment: The affect infusion model (AIM). Psychological Bulletin, 117, 39-66.

Forgas, J. P. (1998). Asking nicely? The effects of mood on responding to more or less polite requests. Personality \& Social Psychology Bulletin, 24, 173-185.

Forgas, J. P. (1999a). Feeling and speaking: Mood effects on verbal communication strategies. Personality \& Social Psychology Bulletin, 25, $850-863$

Forgas, J. P. (1999b). On feeling good and being rude: Affective influences on language use and request formulations. Journal of Personality and Social Psychology, 76, 928-939.

Forgas, J. P., \& Fiedler, K. (1996). Us and them: Mood effects on intergroup discrimination. Journal of Personality and Social Psychology, 70, 28-40.

Gallo, L. C., Bogart, L. M., Vranceanu, A., \& Matthews, K. A. (2005). Socioeconomic status, resources, psychological experiences, and emotional responses: A test of the reserve capacity model. Journal of Personality and Social Psychology, 88, 386-399.

Green, A. S., Rafaeli, E., Bolger, N., Shrout, P. E., \& Reis, H. T. (2006). Paper or plastic? Data equivalence in paper and electronic diaries. Psychological Methods, 11, 87-105.

Greenier, K. D., Kernis, M. H., McNamara, C. W., Waschull, S. B., Berry, A. J., Herlocker, C. E., et al. (1999). Individual differences in reactivity to daily events: Examining the roles of stability and level of self-esteem. Journal of Personality, 67, 185-208.

Haidt, J. (2002). 'Dialogue between my head and my heart': Affective influences on moral judgment. Psychological Inquiry, 13, 54-56.

Hariri, A. R., \& Holmes, A. (2006). Genetics of emotional regulation: The role of the serotonin transporter in neural function. Trends in Cognitive Science, 10, 182-191.

Harvey, A. G., Watkins, E., Mansell, W., \& Shafran, R. (2004). Cognitive 
behavioural processes across psychological disorders: A transdiagnostic approach to research and treatment. New York: Oxford University Press.

Ito, T. A., \& Cacioppo, J. T. (2001). Affect and attitudes: A social neuroscience approach. In J. P. Forgas (Ed.), Handbook of affect and social cognition (pp. 50-74). Mahwah, NJ: Erlbaum.

Kensinger, E. A., \& Schacter, D. L. (2008). Memory and emotion. In M. Lewis, J. M. Haviland-Jones, \& L. F. Barrett (Eds.), The handbook of emotion (3rd ed.). New York: Guilford Press.

Kernis, M. H. (2005). Measuring self-esteem in context: The importance of stability of self-esteem in psychological functioning. Journal of Personality, $73,1-37$

Kring, A. M. (2008). Emotion disturbances as transdiagnostic processes in psychopathology. In M. Lewis, J. M. Haviland-Jones, \& L. F. Barrett (Eds.), The handbook of emotion (3rd ed.). New York: Guilford Press.

Larsen, R. J., Diener, E., \& Emmons, R. A. (1986). Affect intensity and reactions to daily life events. Journal of Personality and Social Psychology, 51, 803-814.

Laurenceau, J. P., Barrett, L. F., \& Pietromonaco, P. R. (1998). Intimacy as a process: The importance of self-disclosure and responsiveness in interpersonal exchanges. Journal of Personality and Social Psychology, $74,1238-1251$.

Messinger, D. S. (2002). Positive and negative: Infant facial expressions and emotions. Current Directions in Psychological Science, 11, 1-6.

Moreno, K. N., \& Bodenhausen, G. V. (2001). Intergroup affect and social judgement: Feelings as inadmissible information. Group Processes and Intergroup Relations, 4, 21-29.

Mosteller, F., \& Bush, R. R. (1954). Selected quantitative techniques. In G. Lindzey (Ed.), Handbook of social psychology (pp. 289-334). Cambridge, MA: Addison-Wesley.

Nezlek, J. B. (2005). Distinguishing affective and non-affective reactions to daily events. Journal of Personality, 73, 1539-1568.

Nezlek, J. B., \& Plesko, R. M. (2003). Affect- and self-based models of relationships between daily events and daily well-being. Personality and Social Psychology Bulletin, 29, 584-596.

Ortony, A., \& Turner, T. J. (1990). What's basic about basic emotions? Psychological Review, 97, 315-331.

Pietromonaco, P. R., \& Barrett, L. F. (1997). Working models of attachment and daily social interactions. Journal of Personality and Social Psychology, 73, 1409-1423.

Quigley, K. S., \& Barrett, L. F. (1999). Emotional learning and mechanisms of intentional psychological change. In R. M. L. J. Brandtstadter (Ed.), Action and development: Origins and functions of intentional self-development (pp. 435-464). Thousand Oaks, CA: Sage.

Raudenbush, S. W., Bryk, A. S., Cheong, Y. F., \& Congdon, R. T. (2004). HLM: Hierarchical linear and nonlinear modeling. Chicago: Scientific Software International.

Reis, H. T., \& Wheeler, L. (1991). Studying social interaction with the Rochester Interaction Record. In M. P. Zanna (Ed.), Advances in exper- imental social psychology (Vol. 24; pp. 269-318). San Diego, CA: Academic Press.

Revelle, W. (1995). Personality processes. Annual Review of Psychology, 46, 295-328.

Rosenberg, M. (1965). Society and the adolescent self-image. Princeton, NJ: Princeton University Press.

Rosenthal, R. (1984). Meta-analytic procedures for social research. Beverly Hills, CA: Sage.

Russell, J. A. (2003). Core affect and the psychological construction of emotion. Psychological Review, 110, 145-172.

Russell, J. A., Bachorowski, J.-A., \& Fernández-Dols, J.-M. (2003). Facial and vocal expressions of emotion. Annual Review of Psychology, 54, 329-349.

Russell, J. A., \& Barrett, L. F. (1999). Core affect, prototypical emotional episodes, and other things called emotion: Dissecting the elephant. Journal of Personality and Social Psychology, 76, 805-819.

Russell, J. A., Weiss, A., \& Mendelsohn, G. A. (1989). Affect grid: A single-item scale of pleasure and arousal. Journal of Personality and Social Psychology, 57, 493-502.

Sargent, J. T., Crocker, J., \& Luhtanen, R. K. (2006). Contingencies of self-worth and depressive symptoms in college students. Journal of Social and Clinical Psychology, 25, 628-646.

Searle, J. (2004). Mind: A brief introduction. New York: Cambridge University Press.

Seo, M., Barrett, L. F., \& Bartuneck, J. M. (2004). The role of affective experience in work motivation. Academy of Management Review, 29, 423-439.

Slovic, P., Finucane, M., Peters, E., \& MacGregor, D. G. (2002). Rational actors or rational fools: Implications of the affect heuristic for behavioral economics. Journal of Socio-Economics, 31, 329-342.

Watson, D. (2000). Mood and temperament. New York: Guilford Press.

Weinstock, L. M., \& Whisman, M. A. (2006). Neuroticism as a common feature of the depressive and anxiety disorders: A test of the Revised Integration Hierarchical Model in a national sample. Journal of Abnormal Psychology, 115, 68-74.

Yik, M. S. M., Russell, J. A., Ahn, C., Dols, J. M. F., \& Suzuki, N. (2002). Relating the five-factor model of personality to a circumplex model of affect: A five language study. In R. R. McCrae \& J. Allik (Eds.), The five-factor model of personality across cultures: International and cultural psychology series (pp. 79-104). New York: Kluwer Academic/ Plenum Press.

Zeigler-Hill, V., \& Showers, C. J. (2007). Self-structure and self-esteem stability: The hidden vulnerability of compartmentalization. Personality and Social Psychology Bulletin, 33, 143-159.

Received March 11, 2008

Revision received January 20, 2009

Accepted January 26, 2009 